\title{
Utero-ovarian ligament fibroid-an unusual location of extrauterine fibroids
}

\author{
Arunima Saini*, Meenakshi Gothwal, Pratibha Singh, Garima Yadav
}

Department of Obstetrics and Gynaecology, AIIMS, Jodhpur, Rajasthan, India

Received: 29 September 2020

Accepted: 05 November 2020

\section{*Correspondence:}

Dr. Arunima Saini,

E-mail: arunima0123@gmail.com

Copyright: (C) the author(s), publisher and licensee Medip Academy. This is an open-access article distributed under the terms of the Creative Commons Attribution Non-Commercial License, which permits unrestricted non-commercial use, distribution, and reproduction in any medium, provided the original work is properly cited.

\begin{abstract}
Utero-ovarian ligament fibroids are among the rarest sites for extrauterine leiomyomas. Broad ligament fibroids are relatively common. They can be either asymptomatic or present with chronic pelvic pain and pressure symptoms. They can be confused with an ovarian mass, broad ligament cyst or a pedunculated fibroid. There are high chances of missing it clinically. Ultrasonography, magnetic resonance imaging (MRI) and computed tomography scan are the imaging techniques used to diagnose such conditions, MRI being the most accurate in ruling out other broad ligament masses with suspected ovarian, tubal or isolated broad ligament cyst. Here is a rare case report of a pedunculated extrauterine leiomyoma with its origin in the utero-ovarian ligament.
\end{abstract}

Keywords: Broad ligament fibroid, Leiomyoma, Utero-ovarian ligament

\section{INTRODUCTION}

Leiomyoma is the most common slow growing benign tumor of smooth muscles. They constitute up to $20-30 \%$ of the reproductive age group. ${ }^{1}$ The commonest site of pelvic leiomyomas is uterus. Leiomyomas rarely arise from extrauterine sites, the most common being broad ligament with an incidence of $<1 \% .^{2}$ Other less common sites include round ligament, ovarian ligament and ovaries. The broad ligament tumors can be either epithelial or mesenchymal, with epithelial tumors being most common. Among the mesenchymal tumors, leiomyoma is the commonest. ${ }^{3}$

It is clinical diagnostic dilemma to differentiate and clearly define the origin of an adnexal mass. It can be either of uterine origin or an ovarian origin. Here, we have an interesting case report as to how it was missed/misdiagnosed on imaging and on clinical assessment.

\section{CASE REPORT}

A 45-year female with para 3, live 3 presented with complaints of a lump in the lower abdomen accompanied with pain in left flank and inguinal region since 15 days. The patient incidentally noticed lump in the suprapubic region approximately measuring the size of a cricket ball. She also complained of increased frequency of micturition, not accompanied by burning, sense of incomplete evacuation or fever. The pain in the left flank and inguinal region was localized, continuous, dull aching with no aggravating or relieving factors. Her menstrual cycle was regular lasting for 4 days coming over 28-30 days, painful, using 2 pads per day with moderate flow. Her vitals and systemic examination were normal. Per-abdomen examination revealed a firm mass corresponding to 16 weeks fetal size with side to side mobility. On speculum examination, the cervix was normal looking, deviated to left and vaginal mucosa was healthy. On vaginum examination, the cervix was firm, deviated to left and the uterus was irregularly enlarged, 
14-16 weeks fetal size, deviated to right with fullness in right fornix, while the left fornix was free. Her haematological and biochemical parameters were normal. Liquid-based cytology was negative for intraepithelial lesion or malignancy. Endometrial biopsy was inconclusive. Ultrasonography pelvis revealed asymmetrically enlarged uterus with multiple heteroechoic well-defined lesions with foci of calcification in myometrium, suggestive of fibroids with largest measuring $6.9 \times 9 \mathrm{~cm}$ in the left lateral wall. Endometrial lining appeared normal and bilateral ovaries were not visualized.

After a clinical and radiological assessment, the patient was planned for total abdominal hysterectomy. Intraoperatively, the uterus was 14 weeks size with multiple subcentimetric subserosal fibroids on anterior wall. A large firm pedunculated mass measuring $8 \times 6 \mathrm{~cm}$ was seen arising from left ovarian ligament. Bilateral tubes and ovaries were grossly normal. Uterovesical fold of peritoneum was dissected and bladder pushed down. Rest of the steps of hysterectomy were done in the usual manner. The specimen was sent for histopathology (Figure 1).

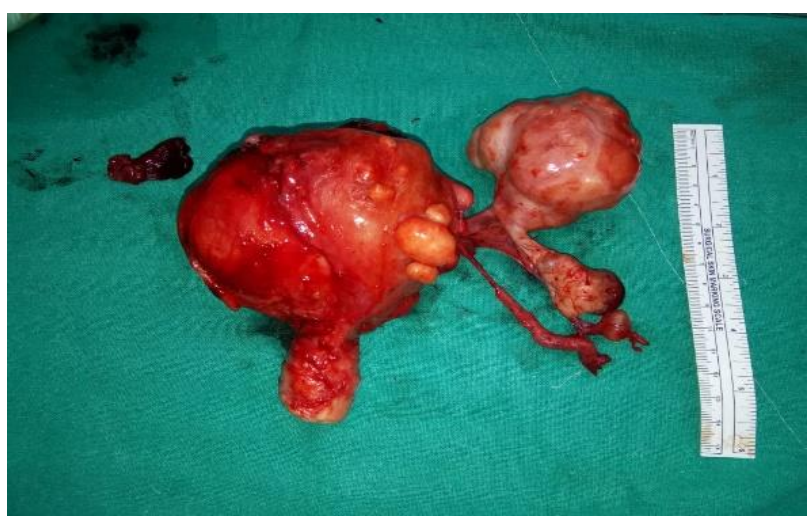

Figure 1: Excised specimen showing multiple anterior wall subserosal fibroids, large pedunculated mass from left utero ovarian ligament.

Grossly, uterus with the cervix measured $14 \times 10 \times 8 \mathrm{~cm}$ and weighed $455.7 \mathrm{gm}$. Uterus contained multiple fibroids, the largest being pedunculated measuring $7 \times 6 \times 5$ $\mathrm{cm}$ with pedicle measuring $3 \times 1 \mathrm{~cm}$. The cut surface was grey-white and whorled. Endometrial thickness was 0.3 $\mathrm{cm}$ and myometrial thickness was $4.5 \mathrm{~cm}$. An intramural fibroid measuring $3.8 \times 3.2 \times 4 \mathrm{~cm}$ with attached left tube $(4$ $\mathrm{cm})$, para tubal cyst and ovary $(3 \times 2 \times 1 \mathrm{~cm})$ was noted.

On microscopic examination, the section from the cervix showed chronic inflammatory infiltrate. Endometrium showed subnuclear vacuolation with stromal oedema. Another section from myometrium showed intersecting bundles of smooth muscles fascicles and areas of extensive hyalinization and thickened blood vessels. Left fallopian tube showed para tubal cyst.

\section{DISCUSSION}

Broad ligament disorders are rare. They remain asymptomatic or give vague complaints unless they get enormously enlarged and cause compression effect thereby presenting with bowel and bladder symptoms. So, there are high chances to miss them clinically. Some conditions of the broad ligament leading to chronic pelvic pain include anatomic defects, rupture of congenital mesonephric cyst causing defects in broad ligament, broad ligament lacerations leading to hypermobility of cervix (Allen master's syndrome), para metritis, posterior broad ligament endometrioma, and benign and malignant tumors. $^{4}$

Leiomyomas are quite common in the reproductive age group of females. They can present with menstrual disturbances, pressure symptoms like bladder and bowel dysfunctions and with infertility or recurrent pregnancy loss if they distort the uterine cavity. Sometimes the myomas undergo secondary changes like degeneration, infarction, necrosis, haemorrhage and very rarely show sarcomatous changes. ${ }^{5}$ Broad ligament leiomyomas are among the commonest extrauterine site where leiomyomas are found. Many case reports have been published where broad ligament fibroid mimics an ovarian mass. ${ }^{6-8}$ Also, there occurs a confusion in differentiating a broad ligament fibroid from a metastatic ovarian carcinoma as it can be associated with pseudoMeigs syndrome causing an elevation of CA 125 levels. Here is a case report of broad ligament leiomyoma arising from utero-ovarian ligament and impinging on the left lateral pelvic wall which was missed both on clinical examination and imaging study.

Differential diagnosis of a broad ligament fibroid includes solid ovarian mass, pedunculated subserosal fibroid, broad ligament cysts or lymphadenopathy. The diagnosis can be made by pelvic examination and imaging techniques like USG, CT scan and MRI. MRI is the most accurate technique for localizing leiomyoma. It is a good modality to differentiate between an ovarian, tubal or a pedunculated fibroid and provides imaging planes that are not available on transabdominal or transvaginal ultrasounds. ${ }^{9}$ Also, secondary changes like degenerations, calcifications, necrosis and sarcoma can be detected by MRI. The final diagnosis is made by histopathology. There are case reports mentioning very massive broad ligament fibroids weighing up to $3.8 \mathrm{~kg} .{ }^{10}$

\section{CONCLUSION}

Broad ligaments fibroids are pelvic masses which can present with vague symptoms or may remain asymptomatic. They can be confused with an ovarian mass clinically and by elevated CA-125 levels. MRI is the imaging modality of choice as the diagnosis may be missed on ultrasonography. Broad ligament is a common site for extrauterine leiomyomas but utero-ovarian ligament is a very rare site for the same. The present case 
is unique as there are few reports of utero-ovarian ligament fibroids in medical literature.

Funding: No funding sources

Conflict of interest: None declared

Ethical approval: Not required

\section{REFERENCES}

1. Kumar P, Malhotra N. Tumours of the corpus uteri and tumours of the pelvic ligament. In: Jeffcoat's Principles of Gynaecology $7^{\text {th }}$ ed. New Delhi: Jaypee Brothers Medical Publishers (P) Ltd. 2008:487-516.

2. Parker WH. Uterine myomas: An overview of development, clinical features, and management. Obstet Gynecol. 2005;105:216-7.

3. Thor AD, Young RH, Clement PB. Pathology of fallopian tube, broad ligament, peritoneum and pelvic soft tissues. Hum Pathol. 1991;22:856-67.

4. Bardawil T, Chelmow D. Broad Ligament Disorders: Overview, Disorders of the Broad Ligament, Broad Ligament Tumors. Available at: https://emedicine.medscape.com/article/275773overview. Accessed on 19 May 2019.

5. Berek JS. Benign diseases of the female reproductive tract. Novack's Gynaecology. $15^{\text {th }}$ ed.
New Delhi: Lippincott Williams and Wilkins, Wolters Kluwer, India. 2007;470.

6. Bansal P, Garg D. A Case of Massive Broad Ligament Leiomyoma Imitating an Ovarian Tumor. J Clin Diagn Res. 2014;8(3):136-7.

7. Kumari R, Vandana, Kulshrestha V, Sharma JB, Kriplani A. A case of broad ligament leiomyoma presenting as an ovarian mass. Int $\mathbf{J}$ Reprod Contracept Obstet Gynecol. 2017;6(6):2635-7.

8. Low SCA, Chong CL. A case of cystic leiomyoma mimicking as ovarian malignancy. Ann Acad Med Singapore. 2004;33:371-4.

9. Fasih N, Shanbhogue PAK, Macdonald DB, FraserHill MA, Papadatos D, Kielar AZ et al. Leiomyomas beyond the uterus: Unusual locations, rare manifestations. Radiograph. 2008;28:1931-48.

10. Bakari F, Sulayman HU, Avidime S, Ameh N, Adesiyun AG. Huge Broad Ligament Leiomyoma: A Case Report. Case Reports in Clin Med. 2015;4:55-9.

Cite this article as: Saini A, Gothwal M, Singh P, Yadav G. Utero-ovarian ligament fibroid-an unusual location of extrauterine fibroids. Int J Reprod Contracept Obstet Gynecol 2020;9:5187-9. 